\title{
EQUIPARTITION OF ENERGY IN LINEARIZED 3-D VISCOELASTICITY
}

\author{
BY \\ GEORGE DASSIOS AND FILARETI ZAFIROPOULOS \\ University of Patras, Patras, Greece
}

\begin{abstract}
A regular and compactly supported initial disturbance propagates in a homogeneous isotropic and linearized viscoelastic medium. The elasticities of the medium exhibit a simple exponential decay in time. General expressions for the energies are obtained and the decay and equipartition of the kinetic and strain energies, for both the longitudinal as well as the transverse wave, are demonstrated.
\end{abstract}

1. Introduction. The idea of equipartition of energy in wave propagation first appeared in the work of Brodsky [2] and independently in the classical book of Lax and Phillips on Scattering Theory [17]. In its definite form, equipartition of energy has been stated first as an asymptotic result by Goldstein [12] and then in finite time by Duffin [10]. Since then a lot of work has been done, as one can find in [13], which contains an almost complete collection of papers in the area. See also [7, 8] and references there. An algebraic approach to equipartition of energy was introduced by Bachelot [1] and Hanouzet [15].

In the present work the energy behaviour of a simple linearized viscoelastic model in a homogeneous and isotropic medium is studied. Rates of decay as well as equipartition of energy results are studied for each one of the longitudinal and the transverse viscoelastic waves. As it is well known, the viscoelastic behaviour of the medium affects the propagation of waves in a rather significant way. In fact, it imposes upon the wave dispersion and attenuation due to the particular viscoelastic constitutive behaviour.

The general approach that we follow in this paper is guided from our previous work in thermoelasticity [7]. Nevertheless, the specific analysis is greatly altered, because of the different dispersion relations governing the propagation of a thermoelastic and a viscoelastic wave. As it is expected, the energy behaviour of a viscoelastic wave is closer to the corresponding behaviour of a purely elastic rather than a thermoelastic wave. General foundations for linear viscoelasticity can be found in references $[4,14]$. Equipartition of energy results for filaments are obtained by Day [9] and Reynolds [19]. 
Section 2 contains a complete statement of the viscoelastic model considered and the Fourier transformation of the governing Cauchy problem. The solution of the transformed problem given in Sec. 2 is provided in Sec. 3, in the form of a Cauchy type integral representation. In Sec. 4 we elaborate on the poles of this representation which are nothing else but the angular frequencies as functions of the wave number. In particular, the low and the high frequency limits are considered in detail. Energy identities, as well as integral representations, for both the longitudinal and the transverse wave, of the kinetic and the strain energy are derived in Sec. 5, while Sec. 6 provides exact form of these integrals and their dependence on time. Section 7 deals with the large time behaviour of the energy integrals. This is a very tedious and long task but only the basic steps are provided. The main conclusions that stem out of the asymptotic analysis of the energy integrals are gathered in the last Sec. 8.

2. The equations of viscoelasticity. Consider the Euclidean space $R^{3}$ occupied by a homogeneous and isotropic material. The material possesses a quiescent past and exhibits viscoelastic behaviour characterized by Lamé constants which fade exponentially $[3,11,18]$. Specifically, we assume the relaxation shear function

$$
\mu:[0,+\infty) \rightarrow R^{+}, \quad \mu(t)=\mu_{\infty}+\mu_{0} e^{-\gamma t}
$$

and the relaxation $\lambda$-function

$$
\lambda:[0,+\infty) \rightarrow R^{+}, \quad \lambda(t)=\lambda_{\infty}+\lambda_{0} e^{-\gamma t}
$$

where $\mu_{\infty}, \lambda_{\infty}, \mu_{0}, \lambda_{0}$, and $\gamma$ are all positive numbers. The constants $\mu_{0}$ and $\lambda_{0}$ represent that part of the relaxation functions that decays to zero as $t \rightarrow+\infty$. The rate of decay is specified by the constant $\gamma$. The assumption of quiescent past allows an extension of the relaxation functions to all of $R$ with vanishing values for $t<0$. Classical elasticity is recovered in the limit as $\gamma \rightarrow 0+$. More general relaxation functions in the form of linear combinations of exponential terms with varying rates of decay [11, p. 287] will make the calculations almost impossible and at the same time will reveal no basic behaviour of the system because it is the smallest rate of decay that will control the propagation of the energy [11, p. 287].

The constitutive relation for linearized viscoelasticity, in our case, assumes the form

$$
\begin{aligned}
\tilde{\boldsymbol{\tau}}(t)= & 2 \mu(0) \tilde{\mathbf{e}}(t)+\lambda(0)(\operatorname{tr} \tilde{\mathbf{e}}(t)) \tilde{\mathbf{I}} \\
& +\int_{0}^{t}[2 \dot{\mu}(s) \tilde{\mathbf{e}}(t-s)+\dot{\lambda}(s)(\operatorname{tr} \tilde{\mathbf{e}}(t-s)) \tilde{\mathbf{I}}] d s
\end{aligned}
$$

where $\tilde{\boldsymbol{\tau}}(t)$ and $\tilde{\mathbf{e}}(t)$ stand for the stress and strain tensors, respectively. Inserting (3) into the Cauchy equation

$$
\rho \ddot{\mathbf{u}}(\mathbf{x}, t)=\nabla \cdot \tilde{\boldsymbol{\tau}}(\mathbf{x}, t)
$$

and expressing everything in terms of the displacement field $\mathbf{u}(\mathbf{x}, t)$ we arrive at the equation of linearized dynamic viscoelasticity

$$
\begin{aligned}
\rho \ddot{\mathbf{u}}(\mathbf{x}, t)= & \mu(0) \Delta \mathbf{u}(\mathbf{x}, t)+(\mu(0)+\lambda(0)) \nabla \nabla \cdot \mathbf{u}(\mathbf{x}, t) \\
& +\int_{0}^{t}[\dot{\mu}(s) \Delta \mathbf{u}(\mathbf{x}, t-s)+(\dot{\mu}(s)+\dot{\lambda}(s)) \nabla \nabla \cdot \mathbf{u}(\mathbf{x}, t-s)] d s .
\end{aligned}
$$


The first two terms in the right-hand side of (5) describe the instantaneous elastic response of the medium, while the integral term provides the effect of the memory from the time the initial disturbance is applied to the present moment $t$.

The initial disturbance is described by the conditions

$$
\begin{aligned}
\mathbf{u}(\mathbf{x}, 0) & =\mathbf{u}_{0}(\mathbf{x}) \\
\dot{\mathbf{u}}(\mathbf{x}, 0) & =\mathbf{u}_{1}(\mathbf{x})
\end{aligned}
$$

where the data $\mathbf{u}_{0}, \mathbf{u}_{1}$ possess enough regularity as the case may be and they are assumed to be compactly supported in $R^{3}$. Equation (5) together with the initial conditions $(6),(7)$ form a well-posed Cauchy problem $[5,14]$ for an integro-partial differential equation, whose solution describes the evolution of an initial disturbance in a homogeneous and isotropic viscoelastic medium.

By means of the spatial Fourier transform

$$
\hat{\mathbf{u}}(\boldsymbol{\xi}, t)=(2 \pi)^{-3 / 2} \int_{R^{3}} e^{i \mathbf{r} \cdot \boldsymbol{\xi}} \mathbf{u}(\mathbf{x}, t) d^{3} x
$$

the Cauchy problem $(5)-(7)$ is reduced to

$$
\begin{aligned}
\rho \ddot{\hat{\mathbf{u}}}(\boldsymbol{\xi}, t)+\left[\mu(0)|\boldsymbol{\xi}|^{2} \tilde{\mathbf{I}}+(\mu(0)+\lambda(0)) \boldsymbol{\xi} \otimes \boldsymbol{\xi}\right] \cdot \hat{\mathbf{u}}(\boldsymbol{\xi}, t) \\
+\int_{0}^{t}\left[\dot{\mu}(s)|\boldsymbol{\xi}|^{2} \tilde{\mathbf{I}}+(\dot{\mu}(s)+\dot{\lambda}(s)) \boldsymbol{\xi} \otimes \boldsymbol{\xi}\right] \cdot \hat{\mathbf{u}}(\boldsymbol{\xi}, t-s) d s=\mathbf{0} \\
\hat{\hat{\mathbf{u}}}(\boldsymbol{\xi}, 0)=\hat{\mathbf{u}}_{0}(\boldsymbol{\xi}) \\
\hat{\hat{\mathbf{u}}}(\boldsymbol{\xi}, 0)=\hat{\mathbf{u}}_{1}(\boldsymbol{\xi})
\end{aligned}
$$

which is an initial value problem for an integro-ordinary differential equation in the time variable alone.

The Fourier variable $\boldsymbol{\xi}$, dual to the position vector $\mathbf{x}$, specifies a plane wave which propagates in the direction

$$
\mathbf{a}=\frac{\boldsymbol{\xi}}{|\boldsymbol{\xi}|},
$$

with the wave number

$$
r=|\xi|
$$

and whose amplitude at $\mathbf{x}$ at the moment $t$ is given by $\mathbf{u}(\mathbf{x}, t)$.

By virtue of (1), (2) and the phase velocities

$$
\left.\begin{array}{rl}
v_{S_{\infty}}^{2}=\frac{\mu_{\infty}}{\rho}, & v_{S_{0}}^{2}=\frac{\mu_{0}}{\rho} \\
v_{P_{\infty}}^{2}=\frac{2 \mu_{\infty}+\lambda_{\infty}}{\rho}, & v_{P_{0}}^{2}=\frac{2 \mu_{0}+\lambda_{0}}{\rho}
\end{array}\right\}
$$

Eq. (9) yields

$$
\begin{aligned}
{[\ddot{\hat{\mathbf{u}}}(t)} & \left.+r^{2}\left(v_{P_{\infty}}^{2}+v_{P_{0}}^{2}\right) \hat{\mathbf{u}}(t)-\gamma r^{2} v_{P_{0}}^{2} e^{-\gamma t} \int_{0}^{t} e^{\gamma / s} \hat{\mathbf{u}}(s) d s\right] \cdot \mathbf{a} \otimes \mathbf{a} \\
& +\left[\ddot{\hat{\mathbf{u}}}(t)+r^{2}\left(v_{S_{\infty}}^{2}+v_{S_{0}}^{2}\right) \hat{\mathbf{u}}(t)-\gamma r^{2} v_{S_{0}}^{2} e^{-\gamma t} \int_{0}^{t} e^{\gamma s} \hat{\mathbf{u}}(s) d s\right] \cdot(\tilde{\mathbf{I}}-\mathbf{a} \otimes \mathbf{a})=\mathbf{0} .
\end{aligned}
$$


The two terms in (15) represent the two orthogonal components of the solution. In particular, projecting (15) into the direction of a we obtain the equation governing the propagation of the longitudinal $(P)$ wave $\hat{\mathbf{u}}^{P}$, while a projection of $(15)$ in the orthogonal to a subspace derives the equation governing the propagation of the transverse $(S)$ wave $\hat{\mathbf{u}}^{S}$. In other words, the form of Eq. (15) affirms the direct sum decomposition of the solution space into a $P$ and an $S$ component subspace because of the dyadic identity

$$
\mathbf{a} \otimes \mathbf{a} \cdot(\tilde{\mathbf{I}}-\mathbf{a} \otimes \mathbf{a})=\tilde{\mathbf{0}} .
$$

In the next section we will obtain an integral representation that solves Eq. (15).

3. Evolution of the $P$ and $S$ waves. In the interest of solving both problems for the longitudinal and the transverse wave simultaneously we introduce the notation

$$
\begin{gathered}
v_{\infty}= \begin{cases}v_{P_{\infty}}, & \text { for the } P \text { wave } \\
v_{S_{\infty}}, & \text { for the } S \text { wave }\end{cases} \\
v_{0}= \begin{cases}v_{P_{0}}, & \text { for the } P \text { wave } \\
v_{S_{0}}, & \text { for the } S \text { wave }\end{cases}
\end{gathered}
$$

and

$$
\hat{\mathbf{U}}(t)=\left\{\begin{array}{ll}
\hat{\mathbf{u}}^{P}(t), & \text { for the } P \text { wave } \\
\hat{\mathbf{u}}^{S}(t), & \text { for the } S \text { wave }
\end{array}\right\} .
$$

Then we have to solve the Cauchy problem

$$
\begin{aligned}
& \ddot{\hat{\mathbf{U}}}(t)+r^{2}\left(v_{\infty}^{2}+v_{0}^{2}\right) \hat{\mathbf{U}}(t)=\gamma r^{2} v_{0}^{2} e^{-i t} \int_{0}^{t} e^{i s} \hat{\mathbf{U}}(s) d s \\
& \left.\begin{array}{rl}
\hat{\mathbf{U}}(0) & =\hat{\mathbf{U}}_{0} \\
\hat{\mathbf{U}}(0) & =\hat{\mathbf{U}}_{1}
\end{array}\right\} \text {. }
\end{aligned}
$$

At the expense of raising the order of differentiation by one, the integration in Eq. (20) can be eliminated, if we multiply through by $e^{i t}$ and differentiate with respect to $t$. This approach leads to the following initial value problem for $\hat{\mathbf{U}}(t)$ :

$$
\begin{aligned}
& \dddot{\mathbf{U}}(t)+\gamma \ddot{\mathbf{U}}(t)+r^{2}\left(v_{\infty}^{2}+v_{0}^{2}\right) \dot{\mathbf{U}}(t)+\gamma r^{2} v_{\infty}^{2} \hat{\mathbf{U}}(t)=\mathbf{0} \\
& \hat{\mathbf{U}}(0)=\hat{\mathbf{U}}_{0} \\
& \dot{\hat{\mathbf{U}}}(0)=\hat{\mathbf{U}}_{1} \\
& \ddot{\hat{\mathbf{U}}}(0)=-r^{2}\left(v_{\infty}^{2}+v_{0}^{2}\right) \hat{\mathbf{U}}_{0} .
\end{aligned}
$$

Using the Laplace transform to solve (22)-(25) we obtain the following integral representation of the solution [16, p. 149, formula $(2.31)]$

$$
\hat{\mathbf{U}}(t)=\frac{1}{2 \pi i} \int_{\Gamma_{r}} \frac{(\tau+\gamma)\left(\tau \hat{\mathbf{U}}_{0}+\hat{\mathbf{U}}_{1}\right) e^{\tau t}}{\tau^{3}+\gamma \tau^{2}+r^{2}\left(v_{\infty}^{2}+v_{0}^{2}\right) \tau+\gamma r^{2} v_{\infty}^{2}} d \tau
$$

where the closed contour $\Gamma_{r}$ encloses all the roots of the polynomial

$$
f(\tau ; r)=\tau^{3}+\gamma \tau^{2}+r^{2}\left(v_{\infty}^{2}+v_{0}^{2}\right) \tau+\gamma r^{2} v_{\infty}^{2}
$$

for every positive value of the wave number $r$. 
In view of the representation (26), the solution of (15) which satisfies the initial conditions (10), (11) assumes the form

$$
\hat{\mathbf{u}}(\boldsymbol{\xi}, t)=\frac{1}{2 \pi i} \int_{\Gamma_{r}}\left(\tau \hat{\mathbf{u}}_{0}(\boldsymbol{\xi})+\hat{\mathbf{u}}_{1}(\boldsymbol{\xi})\right) \cdot\left(\frac{\mathbf{a} \otimes \mathbf{a}}{P(\tau ; r)}+\frac{\tilde{\mathbf{I}}-\mathbf{a} \otimes \mathbf{a}}{S(\tau ; r)}\right)(\tau+\gamma) e^{\gamma t} d \tau,
$$

where

$$
f(\tau ; r)= \begin{cases}P(\tau ; r), & \text { for the } P \text { wave } \\ S(\tau ; r), & \text { for the } S \text { wave }\end{cases}
$$

and $\Gamma_{r}$ encloses the three roots of $P(\tau ; r)$ as well as the three roots of $S(\tau ; r)$. Formula (28) describes the evolution of the transformed viscoelastic field $\hat{\mathbf{u}}$. In particular, the $\mathbf{a} \otimes \mathbf{a}$ term describes the evolution of the longitudinal wave

$$
\hat{\mathbf{u}}^{P}(\boldsymbol{\xi}, t)=\hat{u}^{P}(\boldsymbol{\xi}, t) \mathbf{a}
$$

while the $\tilde{\mathbf{I}}-\mathbf{a} \otimes \mathbf{a}$ term provides the way the transverse wave $\hat{\mathbf{u}}^{S}(\boldsymbol{\xi}, t)$ evolves in time throughout $R^{3}$.

The crucial point in the evolution of the wave field $\hat{\mathbf{u}}(\boldsymbol{\xi}, t)$ is the behaviour of the roots of $P(\tau ; r)$ and $S(\tau ; r)$ in the parameter $r$. In particular, the asymptotic behaviour of the solution for large time depends upon the behaviour of the roots of $P(\tau ; r)$ and $S(\tau ; r)$ for values of $r$ near zero and near infinity [7], i.e., the low and the high frequency response of the wave field.

A further analysis of the solution (28) requires a detailed analysis of the roots of $P(\tau ; r)$ and $S(\tau ; r)$, and forms the content of the next section.

4. A study of the roots of $f(\tau ; r)$. The three roots of the polynomial

$$
f(\tau ; r)=\tau^{3}+\gamma \tau^{2}+r^{2}\left(v_{\infty}^{2}+v_{0}^{2}\right) \tau+\gamma r^{2} v_{\infty}^{2}
$$

are given by [20]

$$
\begin{aligned}
& \tau_{1}(r)=\frac{1}{3}[A+B-\gamma] \\
& \tau_{2}(r)=\frac{1}{3}\left[\omega A+\omega^{2} B-\gamma\right] \\
& \tau_{3}(r)=\frac{1}{3}\left[\omega^{2} A+\omega B-\gamma\right]
\end{aligned}
$$

where

$$
\begin{aligned}
& \omega=\left(\omega^{2}\right)^{*}=-\frac{1}{2}+i \frac{\sqrt{3}}{2} \\
& A=\left[-\gamma^{3}-9 \gamma r^{2} v_{\infty}^{2}+\frac{9}{2} \gamma r^{2} v_{0}^{2}+\frac{3}{2} D^{1 / 2}(r)\right]^{1 / 3} \\
& B=\left[-\gamma^{3}-9 \gamma r^{2} v_{\infty}^{2}+\frac{9}{2} \gamma r^{2} v_{0}^{2}-\frac{3}{2} D^{1 / 2}(r)\right]^{1 / 3}
\end{aligned}
$$

and

$$
D(r)=12 r^{6}\left(v_{\infty}^{2}+v_{0}^{2}\right)^{3}+3 r^{4} \gamma^{2}\left(8 v_{\infty}^{4}-20 v_{\infty}^{2} v_{0}^{2}-v_{0}^{4}\right)+12 r^{2} \gamma^{4} v_{\infty}^{2} .
$$

In particular, if

$$
\frac{v_{\infty}^{2}}{v_{0}^{2}} \geq \frac{5+3 \sqrt{3}}{4} \approx 2.55
$$


then

$$
8 v_{\infty}^{4}-20 v_{\infty}^{2} v_{0}^{2}-v_{0}^{4} \geq 0
$$

and the discriminant $D(r)$ is positive. Condition (39) indicates that the fading out part of the phase velocity should be no more than $62 \%$ of the part of the phase velocity that is due to the instantaneous elastic response of the medium. Because of this condition it is implied that $f$ has the three distinct roots.

$$
\begin{array}{ll}
\tau_{1}(r) \in R, & \forall r \in[0,+\infty) \\
\tau_{2}(r)=\tau_{3}^{*}(r) \in C, & \forall r \in[0,+\infty) .
\end{array}
$$

Our next goal is to obtain asymptotic expansions for the roots of (31) as the parameter (wave number) $r$ approaches zero or infinity. This task demands very long and tedious calculations with the expressions (32)-(38), the Vieta formulae

$$
\begin{gathered}
\tau_{1}(r)+\tau_{2}(r)+\tau_{3}(r)=-\gamma \\
\tau_{1}(r) \tau_{2}(r)+\tau_{2}(r) \tau_{3}(r)+\tau_{3}(r) \tau_{1}(r)=r^{2}\left(v_{\infty}^{2}+v_{0}^{2}\right) \\
\tau_{1}(r) \tau_{2}(r) \tau_{3}(r)=-\gamma r^{2} v_{\infty}^{2}
\end{gathered}
$$

as well as some elementary calculus, essentially as in Sec. 5 in [7]. The results of this analysis are given in the following relations. As $r \rightarrow 0+$

$$
\begin{aligned}
& \tau_{1}(r)=\gamma\left[-1+v_{0}^{2}\left(\frac{r}{\gamma}\right)^{2}+O\left(\left(\frac{r}{\gamma}\right)^{4}\right)\right] \\
& \tau_{2}(r)=\gamma\left[i v_{\infty}\left(\frac{r}{\gamma}\right)-\frac{v_{0}^{2}}{2}\left(\frac{r}{\gamma}\right)^{2}+i \frac{v_{0}^{2} v_{\infty}}{2}\left(1-\frac{v_{0}^{2}}{4 v_{\infty}^{2}}\right)\left(\frac{r}{\gamma}\right)^{3}+O\left(\left(\frac{r}{\gamma}\right)^{4}\right)\right] \\
& \tau_{3}(r)=\gamma\left[-i v_{\infty}\left(\frac{r}{\gamma}\right)-\frac{v_{0}^{2}}{2}\left(\frac{r}{\gamma}\right)^{2}-i \frac{v_{0}^{2} v_{\infty}}{2}\left(1-\frac{v_{0}^{2}}{4 v_{\infty}^{2}}\right)\left(\frac{r}{\gamma}\right)^{3}+O\left(\left(\frac{r}{\gamma}\right)^{4}\right)\right]
\end{aligned}
$$

while as $r \rightarrow+\infty$

$$
\begin{aligned}
& \tau_{1}(r)=\gamma\left[-\frac{v_{\infty}^{2}}{v_{\infty}^{2}+v_{0}^{2}}-\frac{v_{\infty}^{4} v_{0}^{2}}{\left(v_{\infty}^{2}+v_{0}^{2}\right)^{4}}\left(\frac{\gamma}{r}\right)^{2}+O\left(\left(\frac{\gamma}{r}\right)^{4}\right)\right] \\
& \tau_{2}(r)=\gamma\left[i \sqrt{v_{\infty}^{2}+v_{0}^{2}}\left(\frac{r}{\gamma}\right)-\frac{1}{2} \frac{v_{0}^{2}}{v_{\infty}^{2}+v_{0}^{2}}\right. \\
& \left.\quad-i \frac{v_{0}^{4}+4 v_{\infty}^{2} v_{0}^{2}}{8\left(v_{\infty}^{2}+v_{0}^{2}\right)^{5 / 2}}\left(\frac{\gamma}{r}\right)+\frac{v_{\infty}^{4} v_{0}^{2}}{2\left(v_{\infty}^{2}+v_{0}^{2}\right)^{4}}\left(\frac{\gamma}{r}\right)^{2}+O\left(\left(\frac{\gamma}{r}\right)^{3}\right)\right] \\
& \tau_{3}(r)=\gamma\left[-i \sqrt{v_{\infty}^{2}+v_{0}^{2}}\left(\frac{r}{\gamma}\right)-\frac{1}{2} \frac{v_{0}^{2}}{v_{\infty}^{2}+v_{0}^{2}}\right. \\
& \left.+i \frac{v_{0}^{4}+4 v_{\infty}^{2} v_{0}^{2}}{8\left(v_{\infty}^{2}+v_{0}^{2}\right)^{5 / 2}}\left(\frac{\gamma}{r}\right)+\frac{v_{\infty}^{4} v_{0}^{2}}{2\left(v_{\infty}^{2}+v_{0}^{2}\right)^{4}}\left(\frac{\gamma}{r}\right)^{2}+O\left(\left(\frac{\gamma}{r}\right)^{3}\right)\right] .
\end{aligned}
$$

Straightforward calculations lead, in view of the identity

$$
(1-\omega)^{6} \omega^{2}(1+\omega)^{2}=-3^{3},
$$


to the relation

$$
\left(\tau_{1}-\tau_{2}\right)^{2}\left(\tau_{2}-\tau_{3}\right)^{2}\left(\tau_{3}-\tau_{1}\right)^{2}=-\frac{1}{3} D(r) \text {. }
$$

Hence, the expression on the left-hand side of $(53)$ is real and always negative.

We next examine further the dependence of the real root $\tau_{1}(r)$ on $r$ in the whole interval $[0,+\infty)$. To this end we prove that $\tau_{1}$ is a strictly increasing function of the variable

$$
\bar{\rho}=r^{2}
$$

in the open interval $(0,+\infty)$.

First, we observe that $\tau_{1}(\bar{\rho})$ is the one of the three distinct roots of the polynomial

$$
\tau^{3}(\bar{\rho})+\gamma \tau^{2}(\bar{\rho})+\bar{\rho}\left(v_{\infty}^{2}+v_{0}^{2}\right) \tau(\bar{\rho})+\gamma \bar{\rho} v_{\infty}^{2}=0
$$

Therefore, $\tau_{1}$ is an analytic function of $\bar{\rho}$ [16]. Relation (55) is satisfied by the function $\tau_{1}(\bar{\rho})$ for every $\bar{\rho} \in(0,+\infty)$ and an implicit differentiation yields

$$
\left[3 \tau_{1}^{2}(\bar{\rho})+2 \gamma \tau_{1}(\bar{\rho})+\bar{\rho}\left(v_{\infty}^{2}+v_{0}^{2}\right)\right] \tau_{1}^{\prime}(\bar{\rho})+\left[\gamma v_{\infty}^{2}+\left(v_{\infty}^{2}+v_{0}^{2}\right) \tau_{1}(\bar{\rho})\right]=0 .
$$

If there is a $\bar{\rho}_{0} \in(0,+\infty)$ such that

$$
\tau_{1}^{\prime}\left(\bar{\rho}_{0}\right)=0
$$

then (56) concludes

$$
\tau_{1}\left(\bar{\rho}_{0}\right)=-\gamma \frac{v_{\infty}^{2}}{v_{\infty}^{2}+v_{0}^{2}} .
$$

Using mathematical induction we can show, in view of the condition (39), that in fact all the derivatives of $\tau_{1}$ at $\bar{\rho}_{0}$ vanish, i.e.,

$$
\tau^{(n)}\left(\bar{\rho}_{0}\right)=0, \quad n=1,2,3, \ldots
$$

Upon invoking the analyticity of $\tau_{1}(\bar{\rho})$, we expand it in a Taylor series around $\bar{\rho}_{0}$ and conclude, by virtue of (59) that

$$
\tau_{1}(\bar{\rho})=-\gamma \frac{v_{\infty}^{2}}{v_{\infty}^{2}+v_{0}^{2}},
$$

in some neighborhood of $\bar{\rho}_{0}$. This implies that the real root $\tau_{1}$ of (55) is independent of $\bar{\rho}$ in a subinterval of $(0,+\infty)$. Inserting $(60)$ into $(55)$ we obtain

$$
\gamma^{3}\left(\frac{v_{\infty}^{2}}{v_{\infty}^{2}+v_{0}^{2}}-1\right)=0
$$

which implies that $(60)$ is a solution of $(55)$ whenever

$$
\gamma=0
$$

or

$$
v_{0}=0 \text {. }
$$


Both cases (62) and (63) correspond to classical elasticity. Therefore, in viscoelasticity the assumption (57) is false and the real root $\tau_{1}(\bar{\rho})$ is a strictly monotone function of $\bar{\rho}$ in the open interval $(0,+\infty)$. In particular, since

$$
\begin{gathered}
\lim _{\bar{\rho} \rightarrow 0+} \tau_{1}(\bar{\rho})=-\gamma \\
\lim _{\bar{\rho} \rightarrow 0+} \tau_{1}(\bar{\rho})=-\gamma \frac{v_{\infty}^{2}}{v_{\infty}^{2}+v_{0}^{2}}
\end{gathered}
$$

and for the viscoelastic case

$$
\gamma>0, \quad v_{0}>0
$$

we conclude that $\tau_{1}(\bar{\rho})$ is a strictly increasing and always negative function of $\bar{\rho}$.

Evidently, the above behaviour of the real root, as well as the asymptotic expansions (46)-(51) hold true for both the $P(\tau ; r)$ and the $S(\tau ; r)$ polynomials. In the section that follows we derive the necessary expressions for the energy integrals of a viscoelastic wave.

5. The energy of the viscoelastic wave. By virtue of the quiescent past hypothesis and the symmetry property for convolution integrals, Eq. (5) is written as

$$
\begin{aligned}
\rho \ddot{\mathbf{u}}(\mathbf{x}, t)= & \left(\mu_{\infty}+\mu_{0}\right) \Delta \mathbf{u}(\mathbf{x}, t)+\left(\mu_{\infty}+\mu_{0}+\lambda_{\infty}+\lambda_{0}\right) \nabla \nabla \cdot \mathbf{u}(\mathbf{x}, t) \\
& -\mu_{0} \gamma \int_{0}^{t} e^{-\gamma(t-s)} \Delta \mathbf{u}(\mathbf{x}, s) d s \\
& -\left(\mu_{0}+\lambda_{0}\right) \gamma \int_{0}^{t} e^{-\gamma(t-s)} \nabla \nabla \cdot \mathbf{u}(\mathbf{x}, s) d s .
\end{aligned}
$$

In order to obtain an energy identity we multiply (67) by $\dot{\mathbf{u}}(\mathbf{x}, t)$ [5] and utilize the formulae

$$
\begin{aligned}
&(\Delta \mathbf{u}(t)) \cdot \dot{\mathbf{u}}(t)= \nabla \cdot[(\nabla \mathbf{u}(t)) \cdot \dot{\mathbf{u}}(t)]-\frac{\partial}{\partial t}\left[\frac{1}{2}\|\nabla \mathbf{u}(t)\|^{2}\right] \\
&(\nabla \nabla \cdot \mathbf{u}(t)) \cdot \dot{\mathbf{u}}(t)= \nabla \cdot[(\nabla \cdot \mathbf{u}(t)) \dot{\mathbf{u}}(t)]-\frac{\partial}{\partial t}\left[\frac{1}{2}|\nabla \cdot \mathbf{u}(t)|^{2}\right] \\
&(\Delta \mathbf{u}(s)) \cdot \dot{\mathbf{u}}(t)= \nabla \cdot[(\nabla \mathbf{u}(s)) \cdot \dot{\mathbf{u}}(t)] \\
&+\frac{\partial}{\partial t}\left[\frac{1}{2}\|\nabla(\mathbf{u}(t)-\mathbf{u}(s))\|^{2}-\frac{1}{2}\|\nabla \mathbf{u}(t)\|^{2}\right] \\
&(\nabla \nabla \cdot \mathbf{u}(s)) \cdot \dot{\mathbf{u}}(t)= \nabla \cdot[(\nabla \cdot \mathbf{u}(s)) \dot{\mathbf{u}}(t)] \\
&+\frac{\partial}{\partial t}\left[\frac{1}{2}|\nabla \cdot(\mathbf{u}(t)-\mathbf{u}(s))|^{2}-\frac{1}{2}|\nabla \cdot \mathbf{u}(t)|^{2}\right] \\
& \frac{1}{2} \mu_{0} \gamma \int_{-\infty}^{t} e^{-\gamma(t-s)} \frac{\partial}{\partial t}\left(\|\nabla \mathbf{u}(t)\|^{2}\right) d s=\frac{1}{2} \mu_{0} \frac{\partial}{\partial t}\left(\|\nabla \mathbf{u}(t)\|^{2}\right)
\end{aligned}
$$

and

$$
\frac{1}{2}\left(\mu_{0}+\lambda_{0}\right) \gamma \int_{-\infty}^{t} e^{-\gamma(t-s)} \frac{\partial}{\partial t}\left(|\nabla \cdot \mathbf{u}(t)|^{2}\right) d s=\frac{1}{2}\left(\mu_{0}+\lambda_{0}\right) \frac{\partial}{\partial t}\left(|\nabla \cdot \mathbf{u}(t)|^{2}\right)
$$


to derive the conservation law

$$
\begin{aligned}
\frac{\partial}{\partial t}\left\{\frac{\rho}{2}|\dot{\mathbf{u}}(t)|^{2}\right. & +\frac{\mu_{\infty}}{2}\|\nabla \mathbf{u}(t)\|^{2}+\frac{\mu_{\infty}+\lambda_{\infty}}{2}|\nabla \cdot \mathbf{u}(t)|^{2} \\
& \left.+\frac{\gamma}{2} \int_{-\infty}^{t}\left[\mu_{0}\|\nabla(\mathbf{u}(t)-\mathbf{u}(s))\|^{2}+\left(\mu_{0}+\lambda_{0}\right)|\nabla \cdot(\mathbf{u}(t)-\mathbf{u}(s))|^{2}\right] e^{-\gamma(t-s)} d s\right\} \\
= & \cdot\left\{\left(\mu_{\infty}+\mu_{0}\right)(\nabla \mathbf{u}(t)) \cdot \dot{\mathbf{u}}(t)+\left(\mu_{\infty}+\mu_{0}+\lambda_{\infty}+\lambda_{0}\right)(\nabla \cdot \mathbf{u}(t)) \dot{\mathbf{u}}(t)\right. \\
& \left.-\gamma \int_{-\infty}^{t}\left[\mu_{0}(\nabla \mathbf{u}(s)) \cdot \dot{\mathbf{u}}(t)+\left(\mu_{0}+\lambda_{0}\right)(\nabla \cdot \mathbf{u}(s)) \dot{\mathbf{u}}(t)\right] e^{-\gamma(t-s)} d s\right\} .
\end{aligned}
$$

Integrating (74) over the full space $R^{3}$ and using the fact that the support of the initial data is compact we conclude the fundamental energy theorem

$$
E(t)=K(t)+S(t)=E(0), \quad \forall t \in[0,+\infty)
$$

where the kinetic energy is given by

$$
K(t)=\frac{1}{2} \int_{R^{3}} \rho|\dot{\mathbf{u}}(\mathbf{x}, t)|^{2} d^{3} x
$$

and the strain energy by

$$
\begin{aligned}
S(t)= & \frac{1}{2} \int_{\mathbf{R}^{3}}\left[\mu_{\infty}\|\nabla \mathbf{u}(\mathbf{x}, t)\|^{2}+\left(\mu_{\infty}+\lambda_{\infty}\right)|\nabla \cdot \mathbf{u}(\mathbf{x}, t)|^{2}\right] d^{3} x \\
& +\frac{\gamma}{2} \int_{-\infty}^{t} \int_{R^{3}}\left[\mu_{0}\|\nabla(\mathbf{u}(\mathbf{x}, t)-\mathbf{u}(\mathbf{x}, s))\|^{2}\right. \\
& \left.\quad+\left(\mu_{0}+\lambda_{0}\right)|\nabla \cdot(\mathbf{u}(\mathbf{x}, t)-\mathbf{u}(\mathbf{x}, s))|^{2}\right] e^{-\gamma(t-s)} d^{3} x d s .
\end{aligned}
$$

The form of the kinetic energy coincides with the corresponding form in classical elasticity, while the expression for the strain energy involves an extra integral term which accounts for the strain energy of the remaining stress of the material.

Because of the orthogonality between the longitudinal and the transverse wave we can easily show that the total energy of the $P$ wave as well as the total energy of the $S$ wave are also conserved, i.e.,

$$
K^{P}(t)+S^{P}(t)=E^{P}(0), \quad \forall t \in[0,+\infty)
$$

and

$$
K^{S}(t)+S^{S}(t)=E^{S}(0), \quad \forall t \in[0,+\infty) .
$$

By virtue of Parseval's theorem the energies can be expressed in terms of the Fourier transform of the displacement field as

$$
\begin{gathered}
K(t)=\frac{1}{2} \int_{R^{3}} \rho|\dot{\hat{\mathbf{u}}}(\boldsymbol{\xi}, t)|^{2} d^{3} \xi \\
S(t)=\frac{1}{2} \int_{R^{3}}\left[\mu_{\infty}|\hat{\mathbf{u}}(\boldsymbol{\xi}, t)|^{2}+\left(\mu_{\infty}+\lambda_{\infty}\right)|\mathbf{a} \cdot \hat{\mathbf{u}}(\boldsymbol{\xi}, t)|^{2}\right] r^{2} d^{3} \xi \\
+\frac{\gamma}{2} \int_{-\infty}^{t} \int_{R^{3}}\left[\mu_{0}|\hat{\mathbf{u}}(\boldsymbol{\xi}, t)-\hat{\mathbf{u}}(\boldsymbol{\xi}, s)|^{2}\right. \\
\left.+\left(\mu_{0}+\lambda_{0}\right)|\mathbf{a} \cdot(\hat{\mathbf{u}}(\boldsymbol{\xi}, t)-\hat{\mathbf{u}}(\boldsymbol{\xi}, s))|^{2}\right] e^{-\gamma(t-s)} r^{2} d^{3} \xi d s .
\end{gathered}
$$


In the next section we elaborate further on the expressions for the kinetic and the strain energy integrals in order to obtain the exact dependence of these integrals upon the time variable. This step will make the asymptotic analysis of the integrals possible.

6. Expressions for the energy integrals. Let $\tau_{n}^{P}(r), n=1,2,3$ and $\tau_{n}^{S}(r), n=$ $1,2,3$ be the roots of $P(\tau ; r)$ and $S(\tau ; r)$, respectively. Then, the residue theorem of complex analysis can be used to express the solution (28) as

$$
\hat{\mathbf{u}}(\boldsymbol{\xi}, t)=\mathbf{a} \otimes \mathbf{a} \cdot \sum_{n=1}^{3} \mathbf{P}_{n}(\boldsymbol{\xi}) e^{\tau_{n}^{p}(r) t}+(\tilde{\mathbf{I}}-\mathbf{a} \otimes \mathbf{a}) \cdot \sum_{n=1}^{3} \mathbf{S}_{n}(\boldsymbol{\xi}) e^{\tau_{n}^{s}(r) t},
$$

where

$$
\begin{aligned}
\mathbf{P}_{1}(\boldsymbol{\xi}) & =\frac{\left(\tau_{1}^{P}(r)+\gamma\right)}{\left(\tau_{1}^{P}(r)-\tau_{2}^{P}(r)\right)\left(\tau_{1}^{P}(r)-\tau_{3}^{P}(r)\right)}\left[\tau_{1}^{P}(r) \hat{\mathbf{u}}_{0}(r \mathbf{a})+\hat{\mathbf{u}}_{1}(r \mathbf{a})\right] \\
\mathbf{P}_{2}(\boldsymbol{\xi}) & =\frac{\left(\tau_{2}^{P}(r)+\gamma\right)}{\left(\tau_{2}^{P}(r)-\tau_{1}^{P}(r)\right)\left(\tau_{2}^{P}(r)-\tau_{3}^{P}(r)\right)}\left[\tau_{2}^{P}(r) \hat{\mathbf{u}}_{0}(r \mathbf{a})+\hat{\mathbf{u}}_{1}(r \mathbf{a})\right] \\
\mathbf{P}_{3}(\boldsymbol{\xi}) & =\frac{\left(\tau_{3}^{P}(r)+\gamma\right)}{\left(\tau_{3}^{P}(r)-\tau_{1}^{P}(r)\right)\left(\tau_{3}^{P}(r)-\tau_{2}^{P}(r)\right)}\left[\tau_{3}^{P}(r) \hat{\mathbf{u}}_{0}(r \mathbf{a})+\hat{\mathbf{u}}_{1}(r \mathbf{a})\right]
\end{aligned}
$$

and

$$
\begin{aligned}
& \mathbf{S}_{1}(\boldsymbol{\xi})=\frac{\left(\tau_{1}^{S}(r)+\gamma\right)}{\left(\tau_{1}^{S}(r)-\tau_{2}^{S}(r)\right)\left(\tau_{1}^{S}(r)-\tau_{3}^{S}(r)\right)}\left[\tau_{1}^{S}(r) \hat{\mathbf{u}}_{0}(r \mathbf{a})+\hat{\mathbf{u}}_{1}(r \mathbf{a})\right] \\
& \mathbf{S}_{2}(\boldsymbol{\xi})=\frac{\left(\tau_{2}^{S}(r)+\gamma\right)}{\left(\tau_{2}^{S}(r)-\tau_{1}^{S}(r)\right)\left(\tau_{2}^{S}(r)-\tau_{3}^{S}(r)\right)}\left[\tau_{2}^{S}(r) \hat{\mathbf{u}}_{0}(r \mathbf{a})+\hat{\mathbf{u}}_{1}(r \mathbf{a})\right] \\
& S_{3}(\boldsymbol{\xi})=\frac{\left(\tau_{3}^{S}(r)+\gamma\right)}{\left(\tau_{3}^{S}(r)-\tau_{1}^{S}(r)\right)\left(\tau_{3}^{S}(r)-\tau_{2}^{S}(r)\right)}\left[\tau_{3}^{S}(r) \hat{\mathbf{u}}_{0}(r \mathbf{a})+\hat{\mathbf{u}}_{1}(r \mathbf{a})\right] .
\end{aligned}
$$

The actual values of $\tau_{n}^{P}(r), \tau_{n}^{S}(r), n=1,2,3$ are given by (32)-(38) subjected to the notation (17)-(19), as the case may be.

Substitution of (82)-(88) in the energy formulae (80), (81) leads to very long expressions which, after some tedious calculations, can be summarized for each one of the $P$ and the $S$ wave as follows. Incorporate the initial conditions in the notation

$$
\mathbf{W}_{k}(\boldsymbol{\xi})=\left[\tau_{k}(r) \hat{\mathbf{u}}_{0}(\boldsymbol{\xi})+\hat{\mathbf{u}}_{1}(\boldsymbol{\xi})\right] \cdot\left\{\begin{array}{ll}
\mathbf{a} \otimes \mathbf{a}, & \text { for } P \text {-wave } \\
\tilde{\mathbf{I}}-\mathbf{a} \otimes \mathbf{a}, & \text { for } S \text {-wave }
\end{array}\right\}
$$


for $k=1,2,3$, and define the functions

$$
\begin{aligned}
L_{1}(r)= & -\frac{3 r^{2}\left(\tau_{1}+\gamma\right)^{2}\left(\tau_{2}-\tau_{3}\right)^{2}}{D(r)} \int_{|\mathbf{a}|=1}\left|\mathbf{W}_{1}(r \mathbf{a})\right|^{2} d^{2} \mathbf{a} \\
L_{2}(r)= & -\frac{3 r^{2}\left|\tau_{2}+\gamma\right|^{2}\left(\tau_{1}-\tau_{2}\right)\left(\tau_{3}-\tau_{1}\right)}{D(r)} \int_{|\mathbf{a}|=1}\left[\left|\mathbf{W}_{2}(r \mathbf{a})\right|^{2}+\left|\mathbf{W}_{3}(r \mathbf{a})\right|^{2}\right] d^{2} \mathbf{a} \\
L_{3}(r)= & -\frac{3 r^{2}\left(\tau_{2}+\gamma\right)^{2}\left(\tau_{3}-\tau_{1}\right)^{2}}{D(r)} \int_{|\mathbf{a}|=1} \mathbf{W}_{2}(r \mathbf{a}) \cdot \mathbf{W}_{3}^{*}(r \mathbf{a}) d^{2} \mathbf{a} \\
L_{4}(r)= & -\frac{3 r^{2}\left(\tau_{1}+\gamma\right)\left(\tau_{2}+\gamma\right)\left(\tau_{2}-\tau_{3}\right)\left(\tau_{3}-\tau_{1}\right)}{D(r)} \\
& \cdot \int_{|\mathbf{a}|=1}\left[\mathbf{W}_{1}(r \mathbf{a}) \cdot \mathbf{W}_{3}^{*}(r \mathbf{a})+\mathbf{W}_{1}^{*}(r \mathbf{a}) \cdot \mathbf{W}_{2}(r \mathbf{a})\right] d^{2} \mathbf{a} .
\end{aligned}
$$

Then the kinetic energy for each one of the $P$ and $S$ waves is written as

$$
K(t)=K_{1}(t)+K_{23}(t)+K_{2}(t)+K_{12}(t)
$$

where

$$
\begin{aligned}
& K_{1}(t)=\frac{\rho}{2} \int_{0}^{+\infty} \tau_{1}^{2}(r) L_{1}(r) e^{2 \tau_{1}(r) t} d r \\
& K_{23}(t)=\frac{\rho}{2} \int_{0}^{+\infty}\left|\tau_{2}(r)\right|^{2} L_{2}(r) e^{2 \operatorname{Re}\left(\tau_{2}(r)\right) t} d r \\
& K_{2}(t)=\rho \operatorname{Re} \int_{0}^{+\infty} \tau_{2}^{2}(r) L_{3}(r) e^{2 \tau_{2}(r) t} d r \\
& K_{12}(t)=\rho \operatorname{Re} \int_{0}^{+\infty} \tau_{1}(r) \tau_{2}(r) L_{4}(r) e^{\left(\tau_{1}(r)+\tau_{2}(r)\right) t} d r .
\end{aligned}
$$

Similarly, for the strain energy integrals, the quiescent past and the formula

$$
\int_{0}^{t}\left(e^{a t}-e^{a s}\right) e^{-\gamma(t-s)} d s=\frac{a e^{a t}+\gamma e^{-\gamma t}-(a+\gamma) e^{(a-\gamma) t}}{\gamma(a+\gamma)}
$$

imply

$$
S(t)=S_{1}(t)+S_{23}(t)+S_{2}(t)+S_{12}(t)+S_{0}(t)
$$

where

$$
\begin{aligned}
& S_{1}(t)=\frac{\rho}{2} \int_{0}^{+\infty} r^{2}\left[v_{\infty}^{2}+\frac{2 \tau_{1}(r)}{2 \tau_{1}(r)+\gamma} v_{0}^{2}\right] L_{1}(r) e^{2 \tau_{1}(r) t} d r \\
& S_{23}(t)=\frac{\rho}{2} \int_{0}^{+\infty} r^{2}\left[v_{\infty}^{2}+\frac{\tau_{2}(r)+\tau_{3}(r)}{\tau_{2}(r)+\tau_{3}(r)+\gamma} v_{0}^{2}\right] L_{2}(r) e^{2 \operatorname{Re}\left(\tau_{2}(r)\right) t} d r \\
& S_{2}(t)=\rho \operatorname{Re} \int_{0}^{+\infty} r^{2}\left[v_{\infty}^{2}+\frac{2 \tau_{2}(r)}{2 \tau_{2}(r)+\gamma} v_{0}^{2}\right] L_{3}(r) e^{2 \tau_{2}(r) t} d r \\
& S_{12}(t)=\rho \operatorname{Re} \int_{0}^{+\infty} r^{2}\left[v_{\infty}^{2}+\frac{\tau_{1}(r)+\tau_{2}(r)}{\tau_{1}(r)+\tau_{2}(r)+\gamma} v_{0}^{2}\right] L_{4}(r) e^{\left(\tau_{1}(r)+\tau_{2}(r)\right) t} d r
\end{aligned}
$$




$$
\begin{aligned}
S_{0}(t)=\frac{\rho}{2} v_{0}^{2} e^{-\gamma t} & \left\{-\int_{0}^{+\infty} r^{2} L_{1}(r) e^{2 \tau_{1}(r) t} d r\right. \\
& -\int_{0}^{+\infty} r^{2} L_{2}(r) e^{2 \operatorname{Re}\left(\tau_{2}(r)\right) t} d r-2 \operatorname{Re} \int_{0}^{+\infty} r^{2} L_{3}(r) e^{2 \tau_{2}(r) t} d r \\
& -2 \operatorname{Re} \int_{0}^{+\infty} r^{2} L_{4}(r) e^{\left(\tau_{1}(r)+\tau_{2}(r)\right) t} d r+\gamma \int_{0}^{+\infty} \frac{r^{2}}{2 \tau_{1}(r)+\gamma} L_{1}(r) d r \\
& +\gamma \int_{0}^{+\infty} \frac{r^{2}}{2 \operatorname{Re}\left(\tau_{2}(r)\right)+\gamma} L_{2}(r) d r+2 \gamma \operatorname{Re} \int_{0}^{\infty} \frac{r^{2}}{2 \tau_{2}(r)+\gamma} L_{3}(r) d r \\
& \left.+2 \gamma \operatorname{Re} \int_{0}^{+\infty} \frac{r^{2}}{\tau_{1}(r)+\tau_{2}(r)+\gamma} L_{4}(r) d r\right\} .
\end{aligned}
$$

In the interest of obtaining the large time behaviour of the kinetic and the strain energy of the longitudinal and the transverse wave we derive, in the next section, asymptotic expansions for all the integrals appearing in the formulae (94) and (100).

7. Asymptotic expressions of the energy integrals. In order to obtain the asymptotic forms for some of the energy integrals we utilize the following three Lemmas which correspond to Lemmas 3, 7, and 8 in [7], where detailed proofs can be found.

Lemma 1. Consider the integral

$$
F(t)=\int_{0}^{+\infty} r^{k} f(r) e^{-\Phi(r) r^{2} t} d r
$$

for $t>0, k>0$, where

(i) $f(r)$ is a continuous function on $[0,+\infty)$, such that $f(r) \geq 0, f(0) \neq 0$, and $|f(r)|<M_{f}$

(ii)

$$
F(0)=\int_{0}^{+\infty} r^{k} f(r) d r<+\infty ;
$$

(iii) $\Phi(r)$ is a continuous function on $[0,+\infty)$, such that $\Phi(r)>0$;

(iv)

$$
\inf _{r \in[1,+\infty)} \Phi(r) r^{2}=a>0
$$

Then

$$
\lim _{t \rightarrow+\infty} t^{(k+1) / 2} F(t)=\frac{1}{k+1} \frac{f(0)}{(\Phi(0))^{(k+1) / 2}} \int_{0}^{+\infty} e^{-r^{2 \cdot k+1}} d r
$$

Lemma 2. Consider the integral

$$
F(t)=\int_{0}^{+\infty} r^{n} f(r) e^{\left(\tau_{1}(r)+\tau_{2}(r)\right) t} d r
$$

for $t>0, n>0$ and let $g: C \rightarrow C$ be an entire function. Assume that

(i) $f(r)$ is continuous in $[0,+\infty)$ and $|f(r)|<M_{f}$;

(ii) $f(0)=g(0)$; 
(iii) there exists an $R>0$, such that for every $z \in C$ the following estimate holds

$$
|g(z)| \leq \frac{M_{g}}{(1+|z|)^{n+2}} e^{2 R|\operatorname{Im} z|}
$$

(iv)

$$
\int_{0}^{+\infty} r^{n}|f(r)| d r<+\infty
$$

Then

$$
\lim _{t \rightarrow+\infty} t^{(n+1) / 2} F(t)=0 .
$$

LemMa 3. Consider the integral

$$
F(t)=\int_{0}^{+\infty} r^{n} f(r) e^{2 \tau_{2}(r) t} d r
$$

for $t>0, n>0$ and let $g$ be an entire function. Assume the hypotheses (i)-(iv) of Lemma 2. Then

$$
\lim _{t \rightarrow+\infty} t^{(n+1) / 2} F(t)=0 .
$$

Let us examine first the integrals $K_{1}(t), S_{1}(t)$, and $S_{0}(t)$. Since the real root $\tau_{1}(r)$ is an increasing function of $r$, the limits $(64),(65)$ imply the inequalities

$$
-2 \gamma t \leq 2 \tau_{1}(r) t \leq-2 \gamma \frac{v_{\infty}^{2}}{v_{\infty}^{2}+v_{0}^{2}} t
$$

for every $t \in[0,+\infty)$.

Condition (39) and (42) confirm that

$$
L_{1}(r)>0, \quad \forall r \in(0,+\infty) .
$$

Consequently,

$$
C e^{-2 \% t} \leq K_{t}(t), S_{1}(t) \leq C e^{-2 \gamma\left(v_{\infty}^{2} /\left(v_{\infty}^{2}+v_{0}^{2}\right)\right) t}
$$

where $C$ is a known constant.

Inequalities (118) conclude that both integrals $K_{1}(t)$ and $S_{1}(t)$, for either the $P$ or the $S$ wave, exhibit exponential decay with increasing time. A similar sequence of arguments will easily prove that the integral $S_{0}(t)$ is also exponentially decaying.

More precisely, as $t \rightarrow+\infty$,

$$
\begin{gathered}
K_{1}(t)=O\left(e^{-2 \gamma\left(v_{\infty}^{2} /\left(v_{\infty}^{2}+v_{0}^{2}\right)\right) t}\right) \\
S_{1}(t)=O\left(e^{-2 \gamma\left(v_{\infty}^{2} /\left(v_{\infty}^{2}+v_{0}^{2}\right)\right) t}\right) \\
S_{0}(t)=O\left(e^{-\eta t}\right) .
\end{gathered}
$$

Define next the generalized moments

$$
\begin{gathered}
\mathbf{M}_{k}^{P,(n)}(\mathbf{a})=\left.i^{-n} \frac{d^{n}}{d r^{n}}\left[\mathbf{a} \otimes \mathbf{a} \cdot \hat{\mathbf{u}}_{k}(r \mathbf{a})\right]\right|_{r=0} \\
\mathbf{M}_{k}^{S,(n)}(\mathbf{a})=\left.i^{-n} \frac{d^{n}}{d r^{n}}\left[(\tilde{\mathbf{I}}-\mathbf{a} \otimes \mathbf{a}) \cdot \hat{\mathbf{u}}_{k}(r \mathbf{a})\right]\right|_{r=0}
\end{gathered}
$$


for $k=0,1$, and let $v_{0}^{P}, v_{0}^{S}, v_{1}^{P}, v_{1}^{S}$ be the smallest nonnegative integer for which

$$
\begin{aligned}
& \mathbf{M}_{0}^{P,(n)}(\mathbf{a}) \neq \mathbf{0} \\
& \mathbf{M}_{0}^{S,(n)}(\mathbf{a}) \neq \mathbf{0} \\
& \mathbf{M}_{1}^{P,(n)}(\mathbf{a}) \neq \mathbf{0} \\
& \mathbf{M}_{1}^{S,(n)}(\mathbf{a}) \neq \mathbf{0},
\end{aligned}
$$

respectively. Applying Lemma 1 to the integrals $K_{23}(t)$ and $S_{23}(t)$ we obtain, after long but relatively straightforward calculations, the asymptotic relations as $t \rightarrow+\infty$

$$
\begin{aligned}
& K_{23}(t)=H_{m} t^{-(m+(3 / 2))}+O\left(t^{-(m+(5 / 2))}\right) \\
& S_{23}(t)=H_{m} t^{-(m+(3 / 2))}+O\left(t^{-(m+(5 / 2))}\right)
\end{aligned}
$$

where

$$
\begin{gathered}
H_{m}=\frac{1}{2(2 m+3)}\left(\frac{2 \gamma}{v_{0}^{2}}\right)^{m+(3 / 2)}\left(\int_{|\mathbf{a}|=1}|g(\mathbf{a})|^{2} d^{2} \mathbf{a}\right)\left(\int_{0}^{+\infty} e^{-r^{2 /(2 m+3)}} d r\right), \\
m=\min \left\{v_{0}, v_{1}\right\}
\end{gathered}
$$

and $g(\mathbf{a})$ is some function of the initial data.

Finally, Lemma 2 and Lemma 3 can be used to show that

$$
\begin{aligned}
& K_{2}(t)=o\left(t^{-(m+(3 / 2))}\right) \\
& K_{12}(t)=o\left(t^{-(m+(5 / 2))}\right) \\
& S_{2}(t)=o\left(t^{-(m+(3 / 2))}\right) \\
& S_{12}(t)=o\left(t^{-(m+(5 / 2))}\right) .
\end{aligned}
$$

In the study of the asymptotic behaviour of the energy integrals the initial data $\mathbf{u}_{0}$, $\mathbf{u}_{1}$ have been assumed to be as regular as needed. In all cases, it is enough to assume that they belong to $C^{5}$.

8. Decay and equipartition of energy. The asymptotic analysis of the previous section imply the energy decay estimates

$$
\begin{aligned}
& K^{P}(t)=H_{m}^{P} t^{-\left(m^{P}+(3 / 2)\right)}+O\left(t^{-\left(m^{P}+(5 / 2)\right)}\right) \\
& S^{P}(t)=H_{m}^{P} t^{-\left(m^{P}+(3 / 2)\right)}+O\left(t^{-\left(m^{P}+(5 / 2)\right)}\right)
\end{aligned}
$$

for the longitudinal wave, and

$$
\begin{aligned}
& K^{S}(t)=H_{m}^{S} t^{-\left(m^{S}+(3 / 2)\right)}+O\left(t^{-\left(m^{S}+(5 / 2)\right)}\right) \\
& S^{S}(t)=H_{m}^{S} t^{-\left(m^{S}+(3 / 2)\right)}+O\left(t^{-\left(m^{S}+(5 / 2)\right)}\right)
\end{aligned}
$$

for the transverse wave. The upper indices $P, S$ indicate the substitution dictated by (17)-(19). 
Asymptotic equipartition of kinetic and strain energy for each one of the longitudinal and the transverse waves holds in the following sense

$$
\begin{aligned}
& \lim _{t \rightarrow+\infty} \frac{K^{P}(t)}{E^{P}(t)}=\lim _{t \rightarrow+\infty} \frac{S^{P}(t)}{E^{P}(t)}=\frac{1}{2} \\
& \lim _{t \rightarrow+\infty} \frac{K^{S}(t)}{E^{S}(t)}=\lim _{t \rightarrow+\infty} \frac{S^{S}(t)}{E^{S}(t)}=\frac{1}{2} .
\end{aligned}
$$

Obviously, the partial equipartitions (140), (141) imply the global equipartition

$$
\lim _{t \rightarrow+\infty} \frac{K(t)}{E(t)}=\lim _{t \rightarrow+\infty} \frac{S(t)}{E(t)}=\frac{1}{2}
$$

Therefore, the viscoelastic material exhibits the same qualitative behaviour as a classical elastic material [6] but the existence of the memory forces the equipartition to occur asymptotically as time approaches infinity even for compactly supported initial disturbances.

Acknowledgment. The authors acknowledge partial support from the Greek Department of Research and Technology during the preparation of the present work. The first author was also supported form the EEC SCIENCE program under grant number SC1-0079.

\section{REFERENCES}

[1] A. Bachelot, Équipartition de l'Energie pour les Systèmes Hyperboliques et Formes Compatibles, Ann. Inst. Henri Poincaré 46, 45-76 (1987)

[2] R. A. Brodsky, On the asymptotic behaviour of solutions of the wave equation, Proc. Amer. Math. Soc. 18, 207-208 (1967)

[3] R. M. Christensen, Theory of Viscoelasticity. An Introduction, Academic Press, New York, 1982

[4] B. D. Coleman and W. Noll, Foundations of linear viscoelasticity, Rev. Modern Phys. 33, 239-249 (1961)

[5] C. M. Dafermos, Asymptotic stability in viscoelasticity, Arch. Rational Mech. Anal. 37, 297-308 (1970)

[6] G. Dassios, Equipartition of energy in elastic wave propagation, Mech. Res Comm. 6, 45-50 (1979)

[7] G. Dassios and M. Grillakis, Dissipation rates and partition of energy in thermoelasticity, Arch. Rational Mech. Anal. 87, 49-91 (1984)

[8] G. Dassios and M. Grillakis, Asymptotic equipartition rate for wave motion in an even number of space dimensions, J. Math. Anal. Appl. 120, 44-52 (1986)

[9] W. A. Day, The decay of the energy in a viscoelastic body, Mathematica 27, 268-286 (1980)

[10] R. J. Duffin, Equipartition of energy in wave motion, J. Math. Anal. Appl. 32, 386-391 (1970)

[11] A. C. Eringen, Continuum Physics II, Academic Press, New York, 1975

[12] J. A. Goldstein, An asymptotic property of solutions of wave equations, Proc. Amer. Math. Soc. 23, 359-363 (1969)

[13] J. A. Goldstein, A (More-or-Less) Complete Bibliography of Semigroups of Operators Through 1984, Publication of Tulane University, New Orleans, 1984

[14] M. E. Gurtin and E. Sternberg, On the linear theory of viscoelasticity, Arch. Rational. Mech. Anal. 11, 291-356 (1962)

[15] B. Hanouzet, Applications Bilinéaires Compatibles avec un Système à Coefficients Variables. Continuité dans les Espaces de Besov, Comm. Partial Differential Equations 10, 433-465 (1985)

[16] F. John, Partial Differential Equations, 4th edition, Springer-Verlag, New York, 1982 
[17] P. Lax and R. Phillips, Scattering Theory, Academic Press, New York, 1967

[18] M. J. Leitman, The Linear Theory of Viscoelasticity, Handbuch der Physik (S. Flügge, Ed.), Springer-Verlag, Berlin, 1973

[19] D. W. Reynolds, On the equipartition of energy in a linear viscoelastic body, Quart. Appl. Math. 41, 325-336 (1984)

[20] J. V. Uspensky, Theory of Equations, McGraw-Hill, New York, 1948 